\title{
Widespread divergent transcription from prokaryotic promoters
}

\author{
Emily Warman ${ }^{1}$, David Forrest ${ }^{1}$, Joseph T. Wade ${ }^{2,3}$, David C. Grainger ${ }^{1 *}$
}

${ }^{1}$ Institute for Microbiology and Infection, School of Biosciences, University of Birmingham, Edgbaston, Birmingham, B15 2TT, UK

\footnotetext{
${ }^{2}$ Wadsworth Centre, New York State Department of Health, Albany, NY, 12208, USA

${ }^{3}$ Department of Biomedical Sciences, University at Albany, Albany, NY, 12201, USA
}

*for correspondence, d.grainger@ bham.ac.uk Tel: +44 (0)121 4145437 


\begin{abstract}
Promoters are DNA sequences that stimulate the initiation of transcription. In all prokaryotes, promoters are believed to drive transcription in a single direction. Here we show that prokaryotic promoters are frequently bidirectional and drive divergent transcription. Mechanistically, this occurs because key promoter elements have inherent symmetry and often coincide on opposite DNA strands. Reciprocal stimulation between divergent transcription start sites also contributes. Horizontally acquired DNA is enriched for bidirectional promoters suggesting that they represent an early step in prokaryotic promoter evolution.
\end{abstract}

Transcription initiation requires DNA sequences called promoters that interact with RNA polymerase (RNAP) ${ }^{1}$. Promoters consist of ordered core elements with distinct roles ${ }^{2,3}$. For example, most bacterial promoters contain a -10 element that interacts with the housekeeping RNAP $\sigma^{70}$ subunit. This facilitates DNA unwinding ${ }^{4,5}$. In eukaryotes and archaea, the TBP binding TATA box has a similar role ${ }^{6}$. It has long been assumed that promoters are directional, driving transcription in a single orientation determined by promoter element arrangement ${ }^{2,7}$. This view has recently been challenged in eukaryotes ${ }^{8-}$ ${ }^{11}$. Nonetheless, the consensus view is that prokaryotic promoters are unidirectional ${ }^{12}$.

Previous studies have mapped transcription start sites (TSSs) in Escherichia coli by detecting triphosphorylated RNA 5' ends ${ }^{13}$. These TSSs can be assigned to $\sigma^{70}$ binding events identified using ChIP-seq ${ }^{13}$. We noticed that not all $\sigma^{70}$ binding sites were associated with detectable RNA $5^{\prime}$ ends. This was particularly evident for horizontally acquired genes silenced by histone-like nucleoid structuring (H-NS) protein (Figure S1). We reasoned that RNAP might initiate transcription but produce unstable RNAs. To test this, we fused 33 such $\sigma^{70}$ targets to lacZ. Any transcripts produced should be stabilised, and detectable, due to translation. Transcription orientation cannot be directly inferred from $\sigma^{70} \mathrm{ChIP}-$ seq data. Hence, DNA sequences were cloned in both directions (Figure 1a). Surprisingly, over half of the fragments were transcriptionally active in both orientations (Figure 1b). We designated the direction of highest lacZ expression as "forward". On average, "reverse" transcription neared half the "forward" activity (Figure 1c). For a subset of divergent transcript pairs, we mapped RNA 5' ends (Figure 1d). Most reverse TSSs were upstream of the forward TSS and resulted from shared overlapping promoter elements (Figures 1e and S2). Mutations in shared promoter elements (Figure S2) reduced expression in both orientations (Figure 1f).

To understand global patterns of divergent transcription we analysed TSSs independently mapped by RNA 5' polyphosphatase sequencing (PPP-seq), dRNA-seq or cappable-seq ${ }^{13-15}$. In all cases, oppositely orientated TSSs tended to co-locate (Figure 2a). To increase sensitivity, we merged the datasets (Figure 2a, combined). This identified 5,292 divergent TSSs, defined as being separated by between 25 and 7 
bp; $19 \%$ of all detected TSSs in E. coli. We refer to the associated promoters as bidirectional. The most common distance between divergent TSSs was $18 \mathrm{bp}$; transcription initiates either side of overlapping -10 elements (Figure 2a, top expansion). We reasoned that promoter element symmetry must play a major role. To test this, we made a position weight matrix (PWM) describing all E. coli promoter sequences. If the PWM matched adjacent regions of DNA on opposite strands the symmetry score increased. Maximum symmetry correlated with divergent transcription $\left(\mathrm{R}^{2}=0.85\right.$; Figure $2 \mathrm{a}$ bottom expansion). Consistent with this, a DNA sequence logo generated by aligning divergent TSSs, separated by 18 bp, was symmetrical (Figure 2b). Contrastingly, TSSs with no divergent transcript generated an asymmetrical motif (Figure 2c). Note that the first, second and sixth positions of promoter -10 elements (consensus 5'-TATAAT-3') are key for transcription initiation ${ }^{5}$ (Figure 2c). At divergent TSS offset by 18 bp, nucleotides two and six of -10 elements on opposite DNA strands base pair. Hence, these positions are most strongly conserved (Figure $2 b$ ). Example -10 elements arranged in this way are shown in Figure 2d. Divergent transcription also increased at TSSs separated by 29, 23, 12, 10 or 7 bp (Figure S3a). These configurations also correspond to symmetrical base pairing between key -10 element nucleotides, and TSSs, on opposite DNA strands (Figure S3b). The distribution of all bidirectional promoters with respect to genes is shown in Figure 2e.

In E. coli transcription preferentially initiates at an adenine (Figure 2c). For divergent TSSs 18 bp apart, the +1 nucleotide corresponds to position -18 on the opposite DNA strand. Hence, -18 is often a thymine (Figure 2b). A thymine at position -18 can increase transcription by improving interaction between $\sigma^{70}$ residue R451 and the DNA backbone ${ }^{16}$ (Figure 3a). We speculated that the $+1 /-18$ overlap could explain why this configuration is so frequently detected. To test this, we cloned a bidirectional promoter, with $18 \mathrm{bp}$ between TSSs, in both orientations upstream of the $\lambda o o p$ transcriptional terminator (Figure $3 \mathrm{bi}$ ). We also made derivatives where the $A \cdot T$ at each $+1 /-18$ position was replaced with $\mathrm{C} \cdot \mathrm{G}$ (Figure 3 biiiii). We measured RNA synthesis terminated by $\lambda$ oop using in vitro transcription (Figure $3 \mathrm{c}$ ). As expected, altering the TSS reduced production of the associated RNA (compare lane 1 with 5 and 3 with 11); the same mutations also reduced transcription in the opposite direction (compare lane 1 with 9 and 3 with 7). Though $\sigma^{70}$ RA451 was defective at the bidirectional promoters (even lane numbers to 12) it was unimpaired at a control promoter not requiring this contact (lanes 13-14).

To determine the prevalence of bidirectional promoters in bacteria we analysed TSS maps for proteobacteria $^{13-15,17-20}$, actinobacteria ${ }^{21,22}$, and a firmicute ${ }^{23}$. We also mapped TSSs in an additional firmicute, Bacillus subtilis, using cappable-seq (summarised in Figure S4 and Table S1). Co-localised divergent TSSs were abundant in all bacteria analysed (Figure 4a). Proteobacteria and actinobacteria were most similar; divergent TSSs were most frequently separated by 18 or $19 \mathrm{bp}$, and shared a nearidentical symmetrical -10 element with E. coli (Figure S5). Firmicutes used the same range of -10 
element configurations illustrated in Figure S3 for E. coli, albeit with little preference for a single arrangement (Figure S5). For all bacteria, spacing intervals associated with divergent transcription scored highest for symmetry (Figure S5)

Archaeal transcription is closely related to that of eukaryotes; promoters have a TATA box and B recognition element (BRE), located a narrow range of distances from the $\mathrm{TSS}^{24}$. We analysed TSS maps for the archaea Thermococcus kodakarensis and Haloferax volcanii ${ }^{25,26}$. We observed strong signatures of promoter bidirectionality (Figure 4a). In T. kodakarensis, divergent TSSs were predominantly separated by $52 \mathrm{bp}$ and located either side of a shared TATA box element (5'-TTATAAA-3') (Figure $4 b, c$ and S6a). Less frequently, TSSs separated by 36 bp were used (Figures $4 b$ and S6a). Here, the B recognition element (BRE; 5'-CGAAA-3') is positioned so the initial $\mathrm{C} \cdot \mathrm{G}$ bp can also act as the TSS on the opposite DNA strand (Figure 4c). Similar observations were made for $H$. volcanii despite the unusual TATA box consensus (5'-TTWT-3') of haloarchaea (Figure S6b,c). For both species, an independent promoter PWM search identified near identical spacing rules (Figure S6).

Our data demonstrate that divergent transcription from promoters is a process conserved in all life forms. The phenomenon is similarly frequent in diverse prokaryotes (Figure S7) and superficially resembles the situation in eukaryotes. However, the mechanistic basis is fundamentally different (Figure 4d). In eukaryotes, bidirectionality is generated by an activator protein creating two adjacent regions of nucleosome depletion ${ }^{27}$. Thus, divergent TSSs use separate core promoter elements that can be separated by thousands of bp, with no distance optimal. By contrast, divergent transcription in prokaryotes depends on symmetry of key promoter elements; TSSs on opposite strands are closely spaced at preferred intervals. Consequently, for prokaryotes, divergent transcription can be predicted using DNA sequence and recapitulated in vitro with purified components. In eukaryotes, recently acquired DNA is enriched for bidirectional promoters ${ }^{27}$. This has been attributed to pervasive transcription factor binding ${ }^{27}$. We initially identified divergent transcription in horizontally acquired $E$. coli DNA (Figure 1). Furthermore, detection of bidirectional promoters increased in cells lacking $\mathrm{H}$ NS (Figure S8a). This results from elevated promoter frequency and symmetry in foreign genes (Figure S8b). Hence, divergent transcription and promoter evolution are linked in prokaryotes. Strikingly, the proportion of bidirectional promoters used for mRNA production is higher than the equivalent fraction of canonical promoters (Figures 2e, S4d). We conclude that divergent transcription plays a key role in prokaryotic cells. 


\section{FIGURE LEGENDS}

Figure 1: Divergent transcription within horizontally acquired genes. a) $\beta$-galactosidase activity derived from cryptic RNAP binding sites. b) Direction of transcription from cloned DNA fragments. c) Average forward or reverse $\beta$-galactosidase activity of all DNA fragments. d) Start sites mapped by primer extension for selected DNA fragments (orientations labelled a or b). Primer extension products in lanes 1 to 10, sizes in nucleotides (nt). Lanes 11-14 are Maxam-Gilbert sequencing reactions for calibration. e) Schematic representation of core promoter elements associated with divergent transcription. f) Effect of mutating shared core promoter elements.

Figure 2: Widespread divergent transcription from bidirectional promoters in Escherichia coli. a) Heatmaps made using global transcription start site (TSS) data ${ }^{13-15}$ or position weight matrix analysis. TSSs on the top chromosome strand are aligned at the centre of the heatmap (bent arrow, labelled +1 ). Heatmap colour indicates abundance of bottom strand TSSs at that position. The expansion shows the occurrence of bottom strand TSSs in a $50 \mathrm{bp}$ window either side of all top strand promoters. b) Predominant DNA sequence motif associated with bidirectional or c) directional promoters. The xaxis break indicates the variable distance between -10 element and TSS at directional promoters. d) a bidirectional promoter between the E. coli pfs and $d g t$ genes. Promoter -10 elements are bold. TSSs are in uppercase. e) Relative position of all bidirectional E. coli promoters with respect to genes.

Figure 3: Reciprocal stimulation between divergent transcription start sites. a) Structure of RNAP bound to DNA (PDB: 6CA0) ${ }^{28}$. Relevant features labelled. b) DNA templates used for in vitro transcription. For simplicity, only the sequences of promoter -10 elements (labelled) and TSSs (bent arrows) are shown. Opposing DNA strands represented by black or blue lines. Interaction between $\sigma^{70}$ R451 and the DNA backbone indicated by dashes. c) Products of in vitro transcription (using templates in panel b) using either $\sigma^{70}$ or the R451A derivative. The RNAI transcript is derived from the replication origin of the plasmid DNA template.

Figure 4: Bidirectional promoters are widespread in prokaryotes. a,b) Heatmaps indicate abundance and position of TSSs on the bottom DNA strand, relative to the nearest top strand promoter (bent arrow). Species and phylogenetic relationships are indicated to left of heatmaps. c) DNA sequence motifs derived from divergent TSSs in T. kodakarensis. d) Bidirectional promoters have a different basis in prokaryotes and eukaryotes.

\section{REFERENCES}

1. Decker, K. B. \& Hinton, D. M. Transcription regulation at the core: similarities among bacterial, archaeal, and eukaryotic RNA polymerases. Annu. Rev. Microbiol. 67, 113-39 (2013).

2. Browning, D. F. \& Busby, S. J. W. The regulation of bacterial transcription initiation. Nature Reviews Microbiology vol. 2 57-65 (2004).

3. Haberle, V. \& Stark, A. Eukaryotic core promoters and the functional basis of transcription initiation. Nature Reviews Molecular Cell Biology vol. 19 621-637 (2018).

4. Bae, B., Feklistov, A., Lass-Napiorkowska, A., Landick, R. \& Darst, S. A. Structure of a bacterial RNA polymerase holoenzyme open promoter complex. Elife 4, (2015).

5. Feklistov, A. \& Darst, S. A. Structural basis for promoter -10 element recognition by the bacterial RNA polymerase $\sigma$ subunit. Cell 147, 1257-1269 (2011).

6. Kramm, K., Engel, C. \& Grohmann, D. Transcription initiation factor TBP: Old friend new questions. Biochemical Society Transactions vol. 47 411-423 (2019). 
bioRxiv preprint doi: https://doi.org/10.1101/2020.01.31.928960; this version posted February 2 , 2020. The copyright holder for this preprint (which was not certified by peer review) is the author/funder, who has granted bioRxiv a license to display the preprint in perpetuity. It is made available under aCC-BY 4.0 International license.

7. Butler, J. E. F. \& Kadonaga, J. T. The RNA polymerase II core promoter: A key component in the regulation of gene expression. Genes and Development vol. 16 2583-2592 (2002).

8. Core, L. J., Waterfall, J. J. \& Lis, J. T. Nascent RNA sequencing reveals widespread pausing and divergent initiation at human promoters. Science (80-. ). 322, 1845-1848 (2008).

9. $\quad$ Seila, A. C. et al. Divergent transcription from active promoters. Science (80-. ). 322, 1849 1851 (2008).

10. Preker, P. et al. RNA exosome depletion reveals transcription upstream of active human promoters. Science (80-. ). 322, 1851-1854 (2008).

11. He, Y., Vogelstein, B., Velculescu, V. E., Papadopoulos, N. \& Kinzler, K. W. The antisense transcriptomes of human cells. Science (80-. ). 322, 1855-1857 (2008).

12. Browning, D. F. \& Busby, S. J. W. Local and global regulation of transcription initiation in bacteria. Nature Reviews Microbiology vol. 14 638-650 (2016).

13. Singh, S. S. et al. Widespread suppression of intragenic transcription initiation by H-NS. Genes Dev. 28, 214-219 (2014).

14. Thomason, M. K. et al. Global transcriptional start site mapping using differential RNA sequencing reveals novel antisense RNAs in Escherichia coli. J. Bacteriol. 197, 18-28 (2015).

15. Ettwiller, L., Buswell, J., Yigit, E. \& Schildkraut, I. A novel enrichment strategy reveals unprecedented number of novel transcription start sites at single base resolution in a model prokaryote and the gut microbiome. BMC Genomics 17, 199 (2016).

16. Singh, S. S., Typas, A., Hengge, R. \& Grainger, D. C. Escherichia coli $\sigma 70$ senses sequence and conformation of the promoter spacer region. Nucleic Acids Res. 39, 5109-5118 (2011).

17. Papenfort, K., Förstner, K. U., Cong, J. P., Sharma, C. M. \& Bassler, B. L. Differential RNAseq of Vibrio cholerae identifies the VqmR small RNA as a regulator of biofilm formation. Proc. Natl. Acad. Sci. U. S. A. 112, E766-E775 (2015).

18. Gill, E. E. et al. High-throughput detection of RNA processing in bacteria. BMC Genomics 19, (2018).

19. Kröger, C. et al. The primary transcriptome, small RNAs and regulation of antimicrobial resistance in Acinetobacter baumannii ATCC 17978. Nucleic Acids Res. 46, 9684-9698 (2018).

20. Sharma, C. M. et al. The primary transcriptome of the major human pathogen Helicobacter pylori. Nature 464, 250-255 (2010).

21. Cortes, T. et al. Genome-wide Mapping of Transcriptional Start Sites Defines an Extensive Leaderless Transcriptome in Mycobacterium tuberculosis. Cell Rep. 5, 1121-1131 (2013).

22. Jeong, Y. et al. The dynamic transcriptional and translational landscape of the model antibiotic producer Streptomyces coelicolor A3(2). Nat. Commun. 7, (2016).

23. Fan, B. et al. dRNA-Seq Reveals Genomewide TSSs and Noncoding RNAs of Plant Beneficial Rhizobacterium Bacillus amyloliquefaciens FZB42. PLoS One 10, e0142002 (2015).

24. Decker, K. B. \& Hinton, D. M. Transcription Regulation at the Core: Similarities Among Bacterial, Archaeal, and Eukaryotic RNA Polymerases. Annu. Rev. Microbiol. 67, 113-139 (2013).

25. Babski, J. et al. Genome-wide identification of transcriptional start sites in the haloarchaeon Haloferax volcanii based on differential RNA-Seq (dRNA-Seq). BMC Genomics 17, (2016).

26. Jäger, D., Förstner, K. U., Sharma, C. M., Santangelo, T. J. \& Reeve, J. N. Primary 
transcriptome map of the hyperthermophilic archaeon Thermococcus kodakarensis. BMC Genomics 15, 684 (2014).

27. Jin, Y., Eser, U., Struhl, K. \& Churchman, L. S. The Ground State and Evolution of Promoter Region Directionality. Cell 170, 889-898.e10 (2017).

28. Narayanan, A. et al. Cryo-EM structure of Escherichia coli 70 RNA polymerase and promoter DNA complex revealed a role of non-conserved region during the open complex formation. $J$. Biol. Chem. 293, 7367-7375 (2018). 


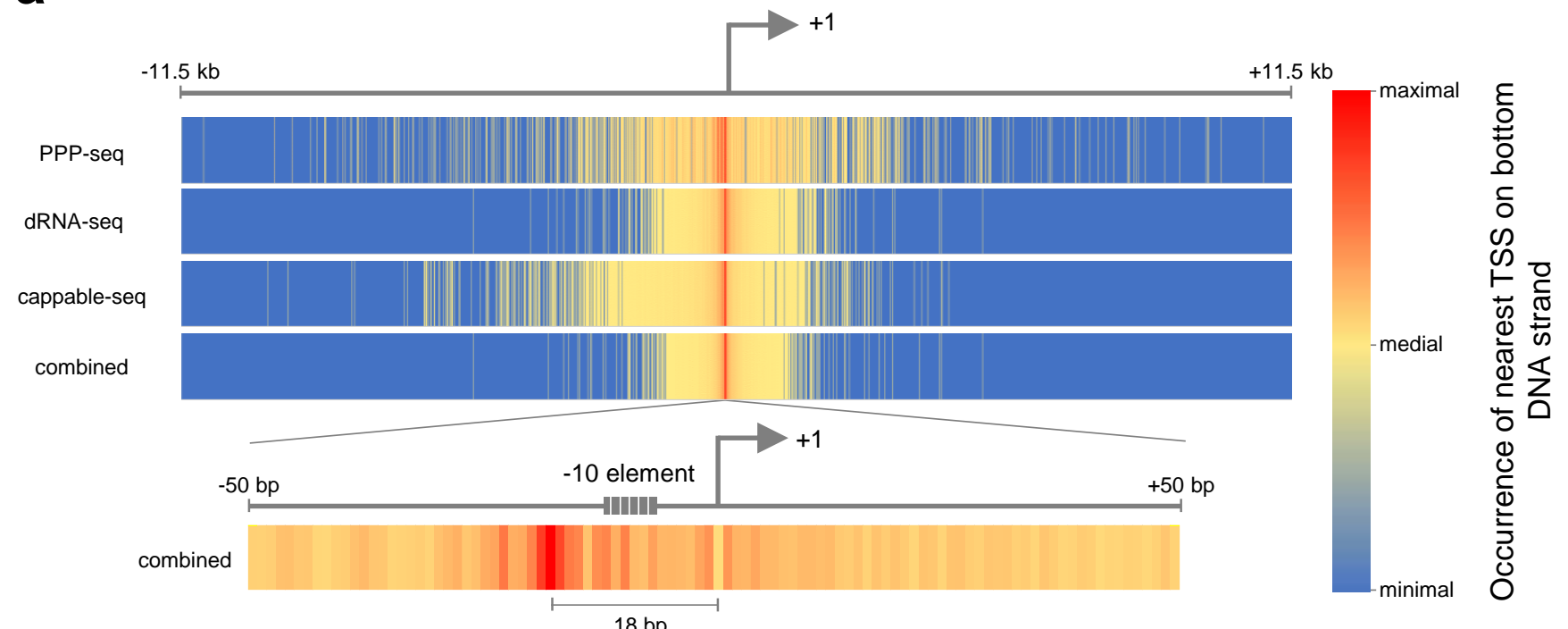

prediction derived from PWM symmetry score

b

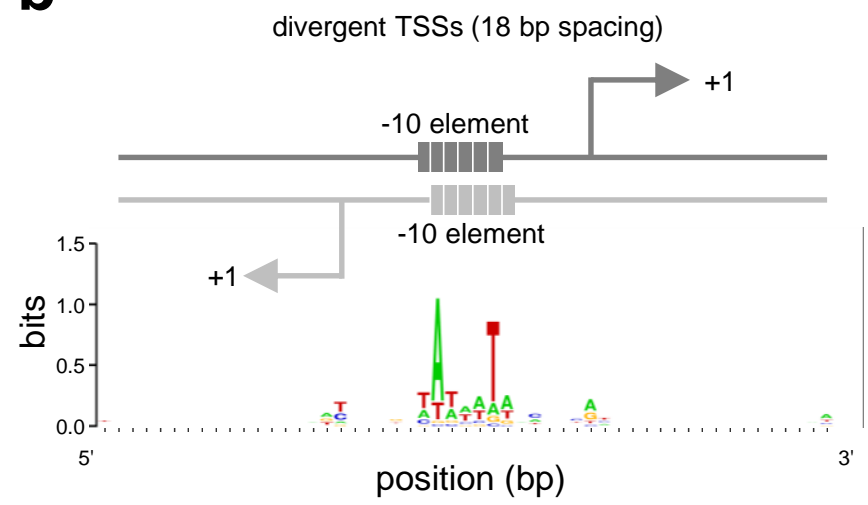

d

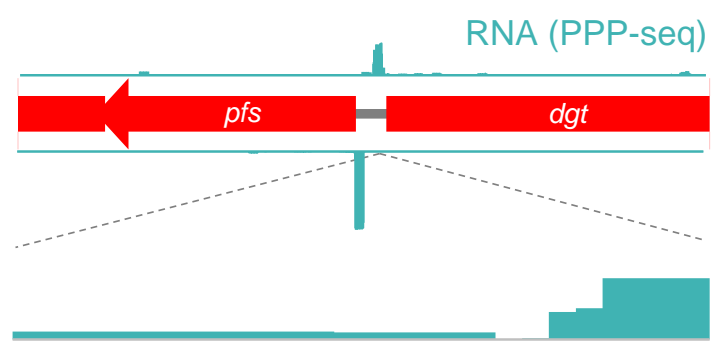

atttgaaggcatagtttaccat $\mathbf{G}$ cgc taaac $\mathbf{T}$ ccgtatcaa
C

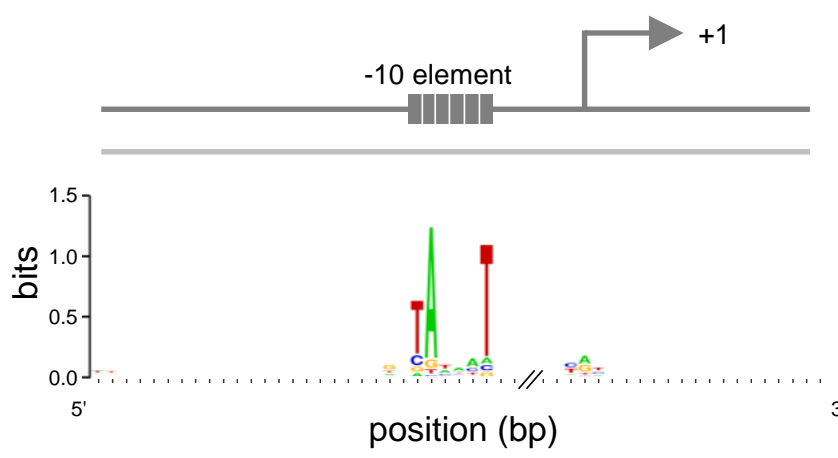

e
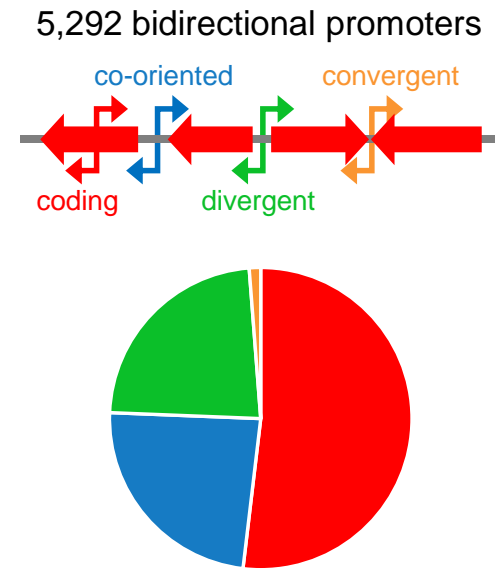
Figure 3

a

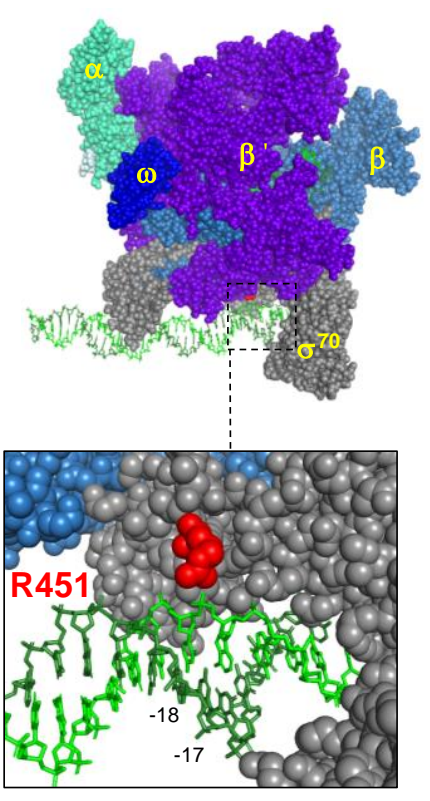

b

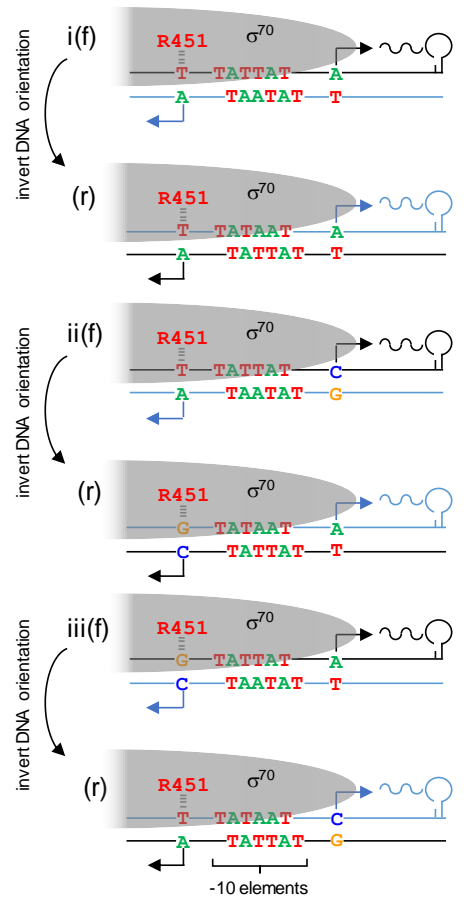

C

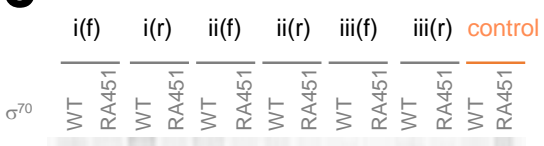

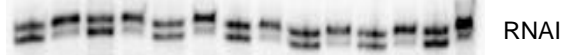




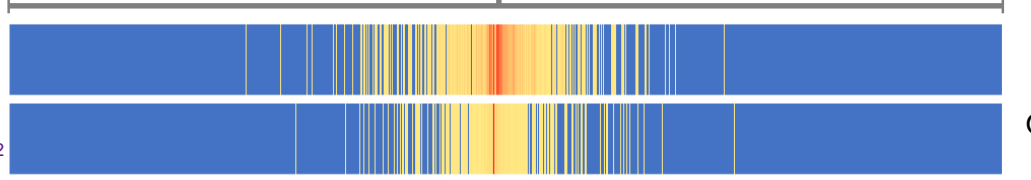

Occurrence of

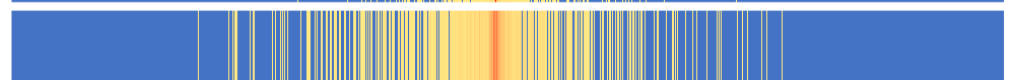
earest TSS

on bottom

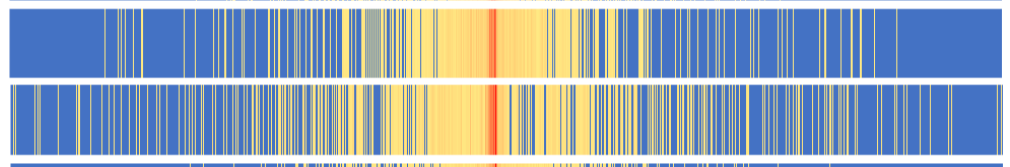

DNA strand

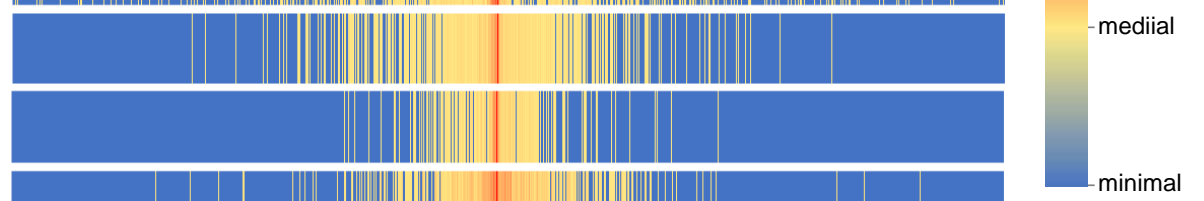

b

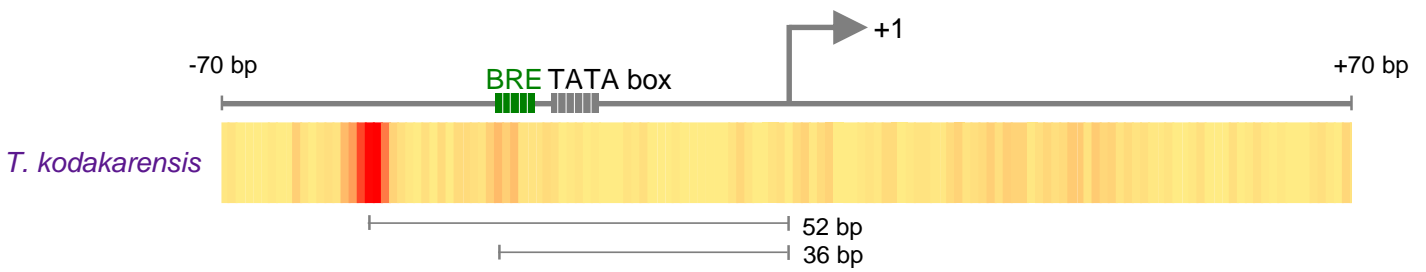

prediction derived from

PWM symmetry score

C

T. kodakarensis divergent TSSs (52 bp spacing)
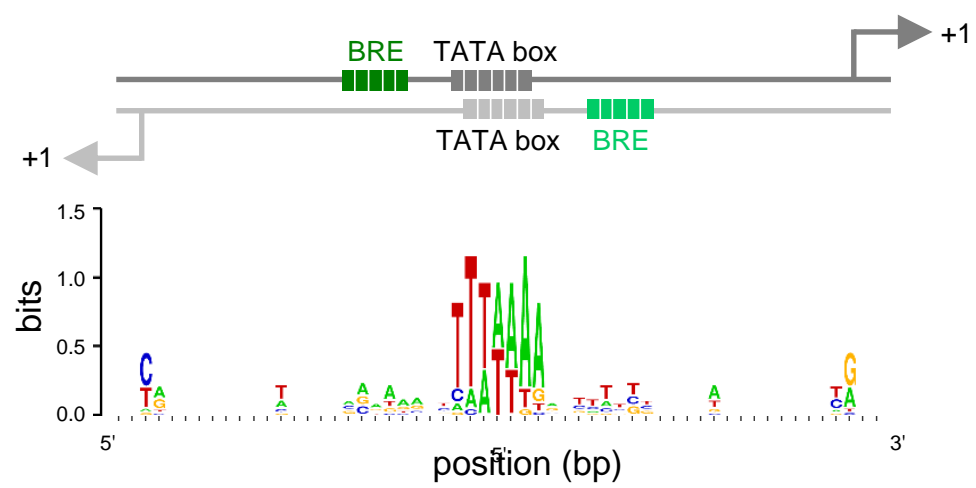

d

\section{prokaryotes}

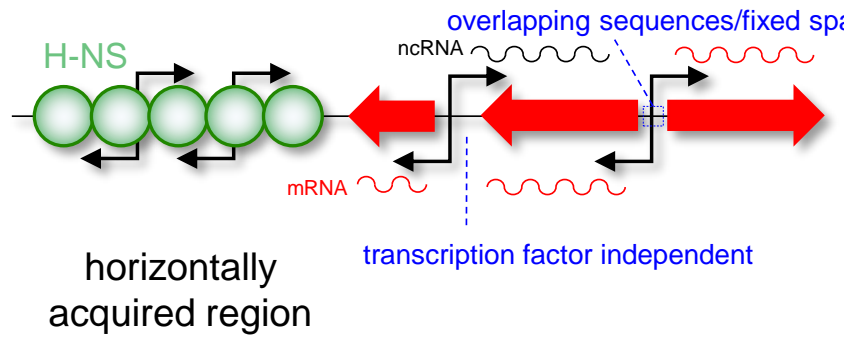

T. Kodakarensis divergent TSSs (36 bp spacing)
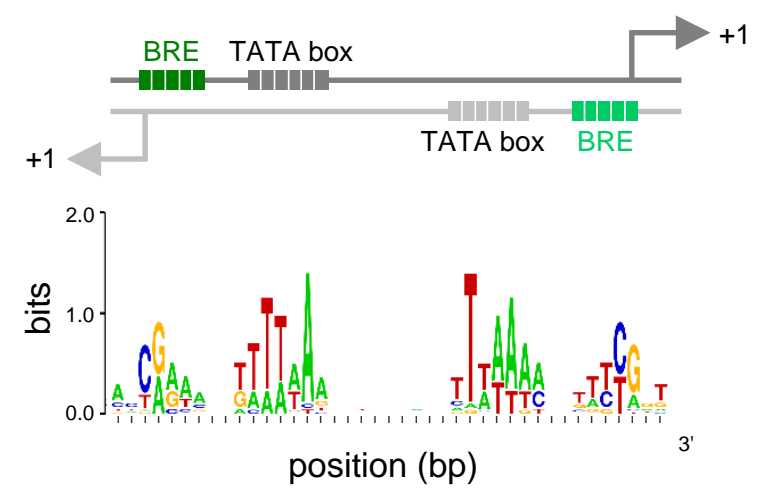

\section{eukaryotes}

independent sequences/variable spacing

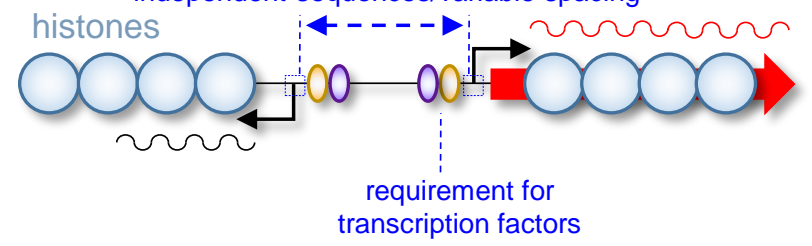

nucleosome depleted/modified region 